\title{
Eva Kocher
}

\section{Tiefe Gefühle im Wettbewerb}

\section{$\mathrm{Zu}$ den Benetton-Entscheidungen« des BGH und des BVerfG}

Im März 2003 hat das Bundesverfassungsgericht zum wiederbolten Mal das zivilgerichtliche Verbot der Benetton-»Schockwerbung " für verfassungwidrig erklärt. Es stellt dabei zu Recht darauf ab, dass auch Werbung, die Gefüble anspreche, eine verfassungsrechtlich geschützte Meinungsäußerung darstellen könne, soweit ibre inhaltliche Intervention ernst gemeint sei und als solche verstanden werde. Einschränkungen der Meinungsäußerungsfreibeit können aber nur zu Gunsten verfassungsrechtlich geschützter Rechtsgüter erfolgen; das Wettbewerbsrecht wird jedenfalls in solchen Werbekontexten küntig deutlicher angeben müssen, welche Rechtsgüter es zu schützen beansprucht. In diesem Zusammenhang ist vor allem der Begriff des Leistungswettbewerbs in Frage zu stellen.

\section{Das Problem: Life-Style-Marketing}

Die »United Colors of Benetton « werben seit jeher für die Kleidung, die sie vertreiben - aber werben sie nicht gleichzeitig auch noch für etwas anderes? Schließlich nennt sich die Marke nicht »Benetton«, sondern »United Colors«; was damit gemeint sein soll, zeigt jede ihrer Kino- und Plakatwerbungen: ${ }^{1}$ Den Kundinnen und Kunden wird ein Identifikationsangebot gemacht: Wer Benetton-Kleidung trägt, ist jung, gibt nichts auf Rassenunterschiede, ist locker und selbstsicher und hat es nicht nötig, Menschen nach ihrer Hautfarbe zu beurteilen.

Dieser Typ Werbung, in dem das Produkt kaum noch präsent ist, ist ein Beispiel für das sogenannte life-style Marketing. ${ }^{2}$ Verkauft wird nicht einfach ein Produkt, sondern eine Lebenshaltung, oder besser: Mit dem Produkt wird über die unmittelbaren Gebrauchs- und Tauschwerte hinaus der Distinktionsgewinn verkauft, den das Produkt in Hinblick auf seine Abgrenzungsfunktion gegenüber anderen Lebenshaltungen verspricht. ${ }^{3}$ Wer möchte heute nicht Teil einer Jugendbewegung sein?4 Heutzutage gibt es jedenfalls unzählige Waren und Dienstleistungen, mit deren Konsum man sich gleichzeitig auch zu einer sozialen Gruppe, einer politischen Haltung oder einer Lebensform bekennt. Das funktioniert auch produktunabhängig - oft kommt es allein auf die Marke und das Image des markenführenden Unternehmens an.

I Das Grundmuster ist zu besichtigen auf der ersten Seite der Homepage auf www.benetton.com.

2 Bruhn/Homburg, Gabler-Marketing-Lexikon, 200I, "Lifestyle-Segmentierung «, "Lebensstil « mit den Hinweisen auf die Strategien der »Marktsegmentierung « und der »Positionierung « von Produkten; ausführlicher Pepels, Marketing, 3. Aufl. 2000, S. 62 ff. zur Marktsegmentierung, S. I I off. zur Positionierung von Produkten und S. I 62 ff. zu life-style Typologien des Käuferverhaltens; vgl. auch zur Werbung als eines Mittels zur Positionierung von Produkten Bergler/Pörzgen/Harich, Frauen und Werbung, I 992, S. I I ff.

3 Der Begriff des Distinktionsgewinns geht auf Bourdieu zurück (vor allem Bourdieu, Die feinen Unterschiede, 8. Aufl. 1996, S. 298 ff. (Drei Arten des Sich-Unterscheidens) und S. 362 ff. (Das Zusammenspiel von Güterproduktion und Geschmacksproduktion).

4 Vgl. Tocotronic, Ich möchte Teil einer Jugendbewegung sein (auf: Tocotronic, Digital ist besser, I995). 
Eine der Bastionen der Kritik an dieser Entwicklung ist der Bundesgerichtshof. Wettbewerb solle »Leistungswettbewerb « sein, und die Leistung bestünde in der Eignung von Produkten für ihre unmittelbaren Gebrauchsfunktionen. ${ }^{5}$ Er hat deshalb immer wieder Werbung für sittenwidrig erklärt, die zum Beispiel hervorhob, dass die beworbenen Produkte in einer Werkstatt für Behinderte hergestellt worden seien, ${ }^{6}$ dass mit dem Kauf eines Hamburgers am »McHappy-Tag « gleichzeitig eine gemeinnützige Organisation unterstützt werde ${ }^{7}$ oder dass der Kauf einem Unternehmen zugute komme, das den Tier- und Artenschutz unterstütze (übrigens unabhängig davon, ob mit dieser Aussage für Sonnenbrillen oder für synthetische Pelze geworben werde). ${ }^{8}$ Die Sittenwidrigkeit ergebe sich daraus, dass diese Werbung nicht sachlich über die Gebrauchseigenschaften des Produkts informiere, sondern die »Gefühle« der Käuferinnen und Käufer anspreche (»gefühlsbetonte Werbung «). ${ }^{9}$

Dabei soll allerdings immer eine Abwägung stattfinden und der Grad der Missbilligung mit dem Grad des Produktbezugs abnehmen. So könne für hand- oder mundgemalte »Künstlerpostkarten « durchaus mit der Körperbehinderung der Künstlerinnen und Künstler geworben werden. ${ }^{\circ}$ Auch bei dem Slogan »Arbeitsplätze bei uns", mit dem ein ostdeutsches Einzelhandelsunternehmen für den Kauf von Waren ostdeutscher Hersteller geworben hatte, war der BGH der Meinung, der sachliche Bezug zwischen dem in der Werbung herausgestellten sozialen Engagement und den beworbenen Produkten sei nicht verloren gegangen. ${ }^{11}$ Mit diesen Kriterien hat sich die Zivilgerichtsbarkeit einen weiten Entscheidungsspielraum offen gehalten.

Die Rechtsprechung über die gefühlsbetonte Werbung hat nun zu einem mit scharfen Worten ausgefochtenen Zusammenstoß zwischen dem Bundesgerichtshof und dem Bundesverfassungsgericht geführt, der erhebliche Auswirkungen auf das gesamte Recht der unlauteren Werbung haben könnte. ${ }^{12}$ Die letzte Entscheidung des Bundesverfassungsgerichts datiert vom März diesen Jahres. Es hat die Sache damit zum zweiten Mal an den Bundesgerichtshof zur Entscheidung zurück verwiesen.

\section{Der Anlass: "Schockwerbung"}

Anlass war eine Werbekampagne der Firma Benetton, mit der sie seit Anfang der 9oer Jahre überregional jeweils zu Beginn der Frühjahr/Sommer- und der Herbst/ Winter-Kollektion ein bis zwei Monate lang in Zeitschriften und an Plakatsäulen warb. Die Kampagne ging auf eine Konzeption des Mailänder Fotografen Oliviero

s Genauer zur rechtlichen Bedeutung des Merkmals unten bei Fn. 65.

6 So wurde wiederholt entschieden, dass Werkstätten für Behinderte in der Werbung für ihre Produkte nicht auf ihren Charakter als Einrichtung nach dem SchwbG hinweisen dürfen (BGH, 22.3. 1967, AP Nr I zu $\$ 9$ SchwBG = GRUR I968, S. 44; BGH, 27. 2. 1980, GRUR I980, S. 800 = NJW I980, S. 2354); Baumbach/ Hefermehl, Wettbewerbsrecht, 22. Aufl. 200I, \ I UWG, Rn I 86c.

7 Vgl. Bülow, Anmerkung zur Entscheidung des BGH vom 7.6. 1995, ZIP I995, S. I 290.

8 So die mit Kammerbeschluss des BVerfG vom 6. 2. 2002, NJW 2002, S. I I 87 ff. aufgehobenen Entscheidungen; vgl. jetzt auch die Entscheidung zur Krombacher-Werbung (Schutz eines Quadratmeter Regenwaldes mit dem Kauf eines Kasten Biers) OLG Hamm, I 2. I I. 2002, NJW 2003, S. I745 ff.

9 Baumbach/Hefermehl (Fn. 6), \ I UWG, RnI75 ff.; Rn I85 ff. zu dieser Fallgruppe; Rn I79 ff. zur umweltbezogenen Werbung und Rn I 87 aff. zur »Schockwerbung «.

I0 BGH, GRUR I959, S. 279; Baumbach/Hefermehl (Fn. 6), \ I UWG, Rn I 86c, anders aber für den Fall, dass ein Unternehmen den Einsatz von Behinderten zu seinem Absatzsystem macht.

I I BGH, 9. 2. I 995, NJW I 995, S. I 964; dazu auch Baumbach/Hefermehl (Fn. 6), \ I UWG, Rn I 86b mit dem Hinweis darauf, dass der Slogan »unauffällig « untergebracht gewesen sei.

I 2 So auch Fezer, Imagewerbung mit gesellschaftskritischen Themen im Schutzbereich der Meinungs- und Pressefreiheit, NJW 200I, S. 58I. 
Toscani zurück und zeigte auf einzelnen Werbebildern etwa eine blutverschmierte

Soldatenuniform; ein Baby unmittelbar nach der Geburt; eine Nonne, die einen Priester küsste; ein überfülltes Flüchtlingsschiff, von dem Menschen ins Meer sprangen; einen elektrischen Stuhl; eine schwarze Frau, die ein weißes Baby stillte; Kinder der Dritten Welt bei schwerer Arbeit; eine ölverschmutzte Ente bzw. ein nacktes menschliches Gesäß, auf das »H.I.V. positive« aufgestempelt war. Am unteren Bildrand befand sich jeweils ein kleines grünes Feld mit dem Schriftzug »United Colors of Benetton«. Beim Deutschen Werberat, einem Organ des Zentralverbands der Deutschen Werbewirtschaft (ZAW), gingen zahlreiche Beschwerden gegen diese »Schockwerbung « ein. ${ }^{13}$ Zahlreiche der Fotos wurden in Kunstausstellungen gezeigt. ${ }^{14}$ Mitte des Jahres 1994 wurde die Werbekampagne in Deutschland gestoppt. Auch in Italien, Frankreich, New York, England, den Niederlanden, Finnland und Norwegen wurden einzelne Plakate verboten - nirgends allerdings so viele wie in Deutschland. ${ }^{\text {is }}$

In den zahlreichen zivilgerichtlichen Auseinandersetzungen in Deutschland obsiegte die Firma Benetton jedenfalls in den Verfahren gegen einige Händler und Händlerinnen, die ihre Franchise-Verträge gekündigt und hohe Umsatzeinbußen geltend gemacht hatten. ${ }^{16}$ Die wettbewerbsrechtliche Auseinandersetzung zieht sich nun jedoch hin. Die Motive »Kinderarbeit«, »ölverschmutzte Ente« und »H.I.V. positive« wurden vom BGH im Jahre I995 zum ersten Mal für wettbewerbswidrig erklärt. ${ }^{17}$ Das zuletzt genannte Motiv war unter anderem Gegenstand einer Klage der Zentrale zur Bekämpfung unlauteren Wettbewerbs gegen die Herausgeberin der Zeitschrift »Stern«, die es veröffentlicht hatte. In diesem Verfahren ergingen die beiden Entscheidungen des Bundesverfassungsgerichts, mit denen jeweils Entscheidungen des Bundesgerichtshofs aufgehoben wurden. ${ }^{18}$

\section{Tiefe Gefüble des Anstands im Wirtschaftsleben}

\section{Die Maßstäbe der Sittenwidrigkeitskontrolle des BGH}

Die Zivilgerichte wandten zunächst die vom BGH entwickelten Kategorien zur Konkretisierung der Generalklausel des \I UWG auf die umstrittene Werbekampagne an. Diese wurde dabei unter anderem mit den Attributen »Aufmerksamkeitswerbung « ${ }^{19}$ und »völlig losgelöst von den Produkten des Unternehmens «² belegt.

I 3 Nach Angaben des ZAW im ersten Verfassungsbeschwerdeverfahren war die Anzahl von 289 Beschwerden die höchste Zahl gegen eine einzige Werbemaßnahme seit Gründung des Deutschen Werberats im Jahre I972 (Stellungnahme des ZAW im Verfassungsgerichtsverfahren, BVerfGE I02, S. 347 ff. (S. 358 )).

I 4 Sevecke, Wettbewerbsrecht und Kommunikationsgrundrechte, I997, S. 24 f, der auch darauf hinweist, dass der Kampagne ein Preis verliehen wurde.

Is Schulze-Fielitz, Anmerkung zur Entscheidung des BVerfG vom I 2. I 2. 2000, JZ 200 I, S. 304; ausführliche Hinweise auf die ausländische »Rechtsprechung « teils der Justiz, teils von Selbstkontrollorganen der Wirtschaft, bei Ahrens, Benetton und Busengrabscher - ein Test für die wettbewerbsrechtliche Sittenwidrigkeitsklausel und die Meinungsfreiheit, JZ ı995, S. I097; siehe auch die Urteile des Marktgerichts Helsinki vom 17. 3. I 1995 und des Marktrats Oslo vom 21. I I. I994, GRUR Int I996, S. 25 I ff.; S. 256 ff. gegen die Verwendung eines Kriegsbildes.

I6 BGH, 8. Zivilsenat, 23. 7. I 997, BGHZ I 36, S. 295 ff.; begründet wurde die Klagabweisung damit, dass die Firma Benetton die Schäden jedenfalls nicht zu vertreten habe; vor Beginn der Werbekampagne habe mit einem Kaufboykott gegen schockierende Werbung nicht gerechnet werden müssen.

I7 BGH, I. Zivilsenat, 6.7. I995, NJW I 995 , S. $2490 \mathrm{ff}$.

I 8 BVerfG, I 2. I 2. 2000, BVerfGE I02, S. 347 ff.; BGH, 6. I 2. 200 I, BGHZ I 49, S. 247 ff.; BVerfG, I I. 3. 2003, ZIP 2003 , S. $642 \mathrm{ff}$.

I9 LG Frankfurt, I6. I2. I993, AfP 1994, S. 242 f.; zum Begriff auch Sevecke (Fn. I4), S. 4I ff.

20 OLG Frankfurt, I3. 8. I992, NJW-RR I993, S. 364 f.; OLG Düsseldorf, 27. 5. I993, WRP I994, S. 69 f. 
Dies allein reichte aber nach einhelliger Meinung noch nicht für ein Verbot aus, ${ }^{2 \mathrm{I}}$ genauso wenig wie das Ärgernis Erregende oder Belästigende, das im Zeigen eines nackten »Körperteils«²2 liegen mochte. Das LG Frankfurt hielt die Werbung mit diesem Foto für zulässig: Die Tatsache, dass die Kundinnen und Kunden »lediglich« dazu veranlasst würden, »sich über das Problem der Aids-Krankheit weitere Gedanken zu machen «, rechtfertige kein Sittenwidrigkeitsurteil. ${ }^{23}$

Mit seiner Einschätzung, die Abbildung rufe beim Betrachter »keine besondere Gefühlsreaktion« hervor, stand es in der zivilgerichtlichen Rechtsprechung aber allein auf weiter Flur. Alle anderen veröffentlichten Urteile gingen davon aus, dass diese Werbung gerade über Gefühle Aufmerksamkeit erregen wolle, also unter die Fallgruppe der "gefühlsbetonten Werbung « falle. Der BGH hielt sie in seinem ersten Urteil vom Juli i 995 jedenfalls unter zwei Aspekten für sittenwidrig:

I) Die Verbindung von »starken Gefühlen des Mitleids und der Ohnmacht [...] und des Schreckens $\ll^{24}$ mit der Werbung sei problematisch (bzw. »makaber «25); hier würden Emotionen zu kommerziellen Zwecken »ausgenutzt«, indem eine Solidarisierung mit dem Namen und der Geschäftstätigkeit des Unternehmens herbeigeführt werde. ${ }^{26}$

2) Die Art der Emotionen und Gedanken, zu denen das Bild »H.I.V. positive« verführten, beeinträchtigten die Menschenwürde der Dargestellten bzw. derer, die sie repräsentierten.

Vor allem das erste, das Ausnutzungs-Argument, geht auf eine lange Tradition in der zivilgerichtlichen Rechtsprechung zur gefühlsbetonten Werbung zurück. Der BGH nahm deshalb sogar an, für den »Stern « sei der »grob wettbewerbswidrige Charakter« auch ohne eine eingehende wettbewerbsrechtliche Prüfung erkennbar gewesen. ${ }^{27}$

\section{Anforderungen der Grundrechterechtsprechung des BVerfG}

Das Bundesverfassungsgericht stellte im Jahre 2000 zunächst klar, dass das BenettonFoto ein »sprechendes Bild mit meinungsbildendem Inhalt « und insofern dem Schutzbereich des Art. 5 GG zuzuordnen sei. ${ }^{28}$ Durch den Werbekontext werde die Ernsthaftigkeit der Botschaft nicht in Frage gestellt; werde sie nicht als ernsthaft verstanden, könne sie schließlich auch kein Mitleid hervorrufen.

2I BGH, 7.6. I995, NJW i995, S. $2490 \mathrm{f}$.

22 Tatbestand des ersten BGH-Urteils vom 6.7. I995, NJW I995, S. 2492 f.

23 LG Frankfurt, I 3. 8. 1993, AfP I994, S. 242 f. Zwei andere Plakate der Reihe wurden aber auch vom LG Frankfurt für sittenwidrig gehalten (WRP i 992, S. 424).

24 BGH, 7.6. I995, NJW I995, S. 2492 f. Für das OLG Frankfurt, I3. 8. 1992, NJW-RR I993, S. 364 f. riefen sie nur »Mitleid und Mitgefühl « hervor; beim Plakat »ölverschmutzte Ente« wurde nach Einschätzung des OLG Düsseldorf, 27. 5. I 993, WRP I 994, S. 69 f. auch die »Angst vor Umweltbedrohungen« mobilisiert.

25 OLG Düsseldorf, 27. 5. I993, WRP ı994, S. 69 f. zum Plakat »ölverschmutzte Ente«; Henning-Bodewig, »Werbung mit der Realität« oder wettbewerbswidrige Schockwerbung, GRUR I993, S. 952.

26 OLG Frankfurt, I3. 8. I992, NJW-RR I993, S. 364 f.; BGH, 6.7. 1995, NJW I 995 , S. 2492 f. Baumbach/ Hefermehl (Fn.6), \ I UWG, Rn i 87aff. stellen maßgeblich auf das Hervorrufen von "Solidaritätsgefühlen«ab.

27 BGH, 7.6. I995, NJW I995, S. 2492 f.; dagegen auch Wassermeyer, Schockierende Werbung, GRUR 2002, S. I3I.

28 Es dürfte mittlerweile herrschende Meinung sein, dass auch Meinungsäußerungen im wirtschaftlichen Wettbewerb dem Schutzbereich unterfallen, wenn die Äußerung über das Anpreisen eines Produkts hinausgeht (dazu sowie zu diesen Grenzen siehe zum Beispiel BVerfG (I. Kammer), 4. I0. I988, NJW I992, S. I I 33 f.; BVerfG (I. Kammer), 27. 5. I 994, NJW I 994, S. 3342 f.; für die zivilgerichtliche Rechtsprechung BGH, I 8. 5. I995, BGHZ I 30, S. 5 ff. (S. I I f.); Ahrens (Fn. I 5), S. I099 ff.; zum Schutzbereich des Art. I0 der Europäischen Menschenrechtskonvention in Bezug auf Äußerungen im wirtschaftlichen Wettbewerb siehe insbesondere EGMR, 23.6. 1994 (Jacubowski ./. Germany), NJW 1995, S. 857 f. 
und dies erforderte die Benennung verfassungsrechtlich geschützter Rechtsgüter, die der Meinungsfreiheit entgegenstehen könnten. Solche ließen sich nach Meinung des Bundesverfassungsgerichts allenfalls gegen ekelerregende, furchteinflößende oder jugendgefährdende Bilder finden. Das Ausnutzungsargument ließe hingegen nicht erkennen, dass der BGH gewichtige öffentliche oder private Rechtsgüter über die »Lauterkeit des Wettbewerbs « gefährdet sah. Das im Verfassungsbeschwerdeverfahren von der Deutschen Vereinigung für gewerblichen Rechtsschutz und Urheberrecht vorgebrachte Argument, das Selbstbestimmungs- und Verbraucherpersönlichkeitsrecht sei beeinträchtigt, ${ }^{29}$ wurde vom Bundesverfassungsgericht mit der Formulierung bedacht: »Ein vom Elend der Welt unbeschwertes Gemüt des Bürgers ist kein Belang, zu dessen Schutz der Staat Grundrechtspositionen einschränken darf. «० Hingegen teilte das BVerfG »selbstverständlich «31 die Auffassung des BGH, dass Werbung, die die Menschenwürde abgebildeter Personen verletze, als sittenwidrig verboten werden könne. Die Begründung, mit der der BGH das Menschenwürdeargument eingesetzt hatte, hatte jedoch keinen Bestand. Dieser hatte in der Anzeige die Darstellung einer »Abstempelung « des HIV-Infizierten und seiner Ausgrenzung aus der menschlichen Gesellschaft gesehen, ${ }^{32}$ ohne sich weiter darum zu bemühen, über die pauschale Behauptung möglicher gesellschaftlicher Abstumpfungstendenzen hinaus zu erläutern, was eine Darstellung von Stigmatisierung, eine Befürwortung von Stigmatisierung und Stigmatisierung als empirische Tatsache im Einzelnen miteinander zu tun hätten.

\section{Sitte und Anstand: normative oder empirische Maßstäbe?}

In Reaktion darauf konzentrierte sich der BGH in seinem nächsten Urteil aus dem Jahre 200I, mit dem er das Sittenwidrigkeitsurteil erneuerte, allein auf das Menschenwürdeargument; diese sei durch die Wirkung der Fotos beeinträchtigt. Nachdem das BVerfG darauf hingewiesen hatte, dass das Bild auch als kritische Stellungnahme zur Stigmatisierung gelesen werden konnte, akzeptiert nun auch der BGH zwar grundsätzlich eine sozialkritische Absicht der Werbung, legt aber dann den Schwerpunkt auf eine Ausdeutung der Art die Gefühle, die durch das Foto hervorgerufen werden könnten. "Bei der wettbewerbsrechtlichen Beurteilung einer Wettbewerbshandlung... kommt es nicht auf die Meinung einer besonders streng urteilenden Minderheit an «.33 Über Mitleid, Ohnmacht und Schrecken hinaus seien auch Reaktionen von Furcht, Angst, Abgestoßensein, Trauer, Bestürzung oder einer tiefgreifenden Verunsicherung möglich: »Alles, was der Betrachter mit der Krankheit Aids verbindet, wird ... mit starker Reizwirkung angesprochen. ... Der Betrachter [wird] ganz seinen

29 Das Argument geht zurück auf Henning-Bodewig, Schockierende Werbung, WRP i992, S. 535; dies. (Fn. 25), S. 952, die Benetton ein »Eindringen « in eine »geistige und emotionale Privatsphäre « vorgeworfen hatte; Kassebohm, Grenzen schockierender Werbung, I995, S. 95 ff., S. I 45 übernimmt zwar ihren Begriff »Schockwerbung «, verwendet ihn aber als »eigenständige Kategorie« (deren Schutzgut er allerdings ebenfalls an keiner Stelle erläutert); auch Sevecke (Fn. I4), S. 62 ff. meint, hier sei eine neue Fallgruppe geschaffen worden (die er kritisiert); S. I 44 ff. kritisch zum »Verbraucherpersönlichkeitsrecht «. In dieselbe Richtung geht die Aussage des BGH (BGH, 6. I 2. 200 I, BGHZ I44, S. 247 ff. (S. 259)), der Schutz des lauteren Wettbewerbs solle auch verhindern, dass ein Spiel mit dem getrieben werde, » was vielen heilig ist «.

30 BVerfG, I 2. I 2. 2000, BVerfGE I02, S. 347 ff.

3 I Schulze-Fielitz (Fn. I s), S. 33 .

32 Die Bewertung als »Abstempelung « wird geteilt von Wehlau, Wettbewerbsrechtliches Gebot zur Achtung der Menschenwürde, DZWIR I996, S. I 44.

$33 \mathrm{BGH}$, 6. I 2. 200 I, BGHZ I 49 , S. $247 \mathrm{ff}$. 
eigenen Assoziationen, Gedanken und Empfindungen überlassen.«Die Wirkung der Anzeige könne je nach Deutung des Fotos und der mit ihm verfolgten Absichten, aber auch je nach eigenem Standpunkt und Lebenssituation des Betrachters sehr verschieden sein.

In diesem Urteil ist vor allem der Aufwand interessant, den der BGH auf die Interpretation des Aussagegehalts und möglicher Wirkungen des Bildes verwendet. Ausführlich geht er auf die Bedeutung unterschiedlicher Vorverständnisse, Beurteilungen, Haltungen und Lebenssituationen (wie etwa ein persönlicher Bezug zu H.I.V.-Infizierten) der Betrachterinnen und Betrachter ein. ${ }^{34}$ Das Bundesverfassungsgericht sollte später - nämlich in dem Urteil aus diesem März, in dem es die Entscheidung wieder aufhob - darauf hinweisen, dass diese Vorgehensweise nicht besonders bemerkenswert sei: »Vom sonst üblichen Vorgang der Ermittlung des Sinns von Äußerungen [aus dem Empfängerhorizont] unterscheidet sich dieses Vorgehen nur terminologisch «.35 Es übersieht aber, dass eine solche sozio-kulturell informierte Berücksichtigung möglicher Empfängerhorizonte in der durchschnittlichen Zivilrechtsprechung durchaus nicht die Regel ist; hier setzt sich der BGH einmal nicht nur formelhaft mit der gesellschaftlichen Wirklichkeit auseinander, sondern bemüht sich um ein Verständnis der Welten realer Verbraucherinnen und Verbraucher in einer gewissen Vielfalt. Die Bestimmung des Empfängerhorizonts bekommt so einen stärker empirischen Gehalt.

Diese Vorgehensweise deckt auch eine der Verständnisschwierigkeiten zwischen BGH und Bundesverfassungsgericht auf: Herkömmlich wird der Begriff der guten Sitten als »Anstandsgefühl « beschrieben; im Rahmen des BGB als »Anstandsgefühl aller billig und gerecht Denkenden «, ${ }^{36}$ im Rahmen des Wettbewerbsrechts als »Anstandsgefühl des verständigen Durchschnittsgewerbetreibenden «37 bzw. als »Anstandsgefühl der beteiligten Verkehrskreise «. $3^{8}$ Dieser Verweis ist auch nach Auffassung des BGH nicht als Verweis auf empirische Wertvorstellungen zu verstehen. Der Begriff der guten Sitten werde in den letzten Jahrzehnten zunehmend normativ verstanden; der Verweis auf »Anstandsgefühle« habe insofern nur »symbolhafte« Bedeutung. Empirische Feststellungen seien nur erforderlich, wo es darum gehe, wie die Werbemaßnahme in den angesprochenen Verkehrskreisen aufgefasst werden könne; hierauf habe die rechtliche Wertung »im Tatsächlichen « »aufzubauen «. Nimmt man diese Aussagen ernst, so dürften die Aussagen zum empirischen Verständnis der Benetton-Anzeige in der angesprochenen Öffentlichkeit allein dazu dienen, mögliche Bedeutungen der Werbeaussage auszuloten, sie also nach einem empirischen Empfängerhorizont auszulegen. Die Bewertung der Werbung habe sodann aber an Hand normativer Maßstäbe zu erfolgen, die der Rechtsordnung, insbesondere den Wertprinzipien der Verfassung zu entnehmen wären (»unter Abwägung der schutzwürdigen Interessen und Güter der Verkehrsbeteiligten und unter Berücksichtigung vorhandener Sozialnormen). ${ }^{39}$

34 Vgl. auch Röhl, Das Recht nach der visuellen Zeitenwende, JZ 2003, S.34I zur Vieldeutigkeit der Kommunikation über Bilder und deren emotionalisierender Wirkung (mit vielen weiteren Nachweisen hierzu). Kassebohm (Fn. 29), S. 5off. bezieht sich schlicht auf öffentliche Stellungnahmen und Umfrageergebnisse zur Benetton-Werbung und meint, so deren Wirkung ergründen zu können.

35 BVerfG, I I. 3. 2003, ZIP 2003, S. 642 ff.; Busche, LM Nr. 866 zu \ I UWG hegt den Verdacht, dass sich der BGH durch die ausführliche Beschäftigung mit dem Aussagegehalt der verfassungsrechtlichen Behandlung entziehe und der Anzeige gerade wegen ihrer Verständnisoffenheit die meinungsbildende Wirkung absprechen wolle.

36 Palandt/Heinrichs, BGB, 62. Aufl. 2003, \I 38 BGB, Rn 2.

37 Baumbach/Hefermehl (Fn. 6), Einl UWG, Rn $86 \mathrm{f}$.

38 BGH, i 8. 5. 1995, BGHZ I30, S. 5 ff. (S. 8).

39 BGH, 7.6. 1995, NJW I995, S. 2492 f. Ausführlich zu diesem Verhältnis empirischer und normativer Elemente Baumbach/Hefermehl (Fn. 6), Einl UWG, Rn 85 ff.; siehe auch schon Teubner, Standards und 
Abgesehen davon, dass damit für diese Bestimmung des normativen Maßstabs doch wieder auf empirische Wertvorstellungen (»Sozialnormen«) verwiesen wird, hält sich der BGH in der Anwendung dieser Grundsätze selbst nicht an seine Postulate. Schon in seiner ersten Benetton-Entscheidung hatte er einen Beweisantrag, der wohl auf Feststellung der empirischen Bildwirkung gerichtet war, missverstanden und es akzeptiert, dass er mit der Begründung abgelehnt worden war, normative Maßstäbe seien nicht tatsächlich zu ermitteln..$^{\circ}$ In der zweiten Entscheidung setzt sich dieses Missverständnis fort, nun allerdings mit der umgekehrten Konsequenz: Er füllt hier die Begriffe des Anstandsgefühls und der Menschenwürde mit empirisch auffindbaren Verständnissen auf. ${ }^{4}$ Die von ihm ebenfalls erwähnten negativen Stellungnahmen H.I.V.-infizierter Personen ${ }^{42}$ sind für den BGH dabei letztlich allerdings nicht entscheidend. Entscheidend wird vielmehr: »Ein Aufruf zur Solidarität mit Menschen in Not ist zynisch, wenn er mit dem Geschäftsinteresse verbunden wird. ... Dieser Zynismus wird noch mehr von denjenigen empfunden werden, die nach ihrer Lebenserfahrung davon ausgehen, dass Wirtschaftswerbung [... bezweckt], die Kunden zu beeinflussen, um sie bereit zu machen, Waren oder Dienstleistungen abzunehmen, und die deshalb ernsthaft an der Aufrichtigkeit eines [...] Aufrufs zur Solidarität mit Aids-Kranken zweifeln.« So endet die ausführliche Beschreibung möglicher Wirkungen der Anzeige in der Behauptung, »entscheidend « hänge die Wirkung der Anzeige davon ab, »wie stark ihr Charakter als Unternehmenswerbung mit gesehen und empfunden wird.«Die Menschenwürde sei also nicht wegen des (komplexen) Aussagegehalts der Anzeige verletzt, sondern weil die Anzeige wegen des kommerziellen Zusammenhangs »in der Öffentlichkeit « » weit überwiegend «als Menschenwürdeverletzung angesehen werde.43

\section{Besondere Anforderungen an Meinungsäußerungen im Wettbewerb?}

Diese Verknüpfung ermöglicht es dem BGH nun auch, das Argument der Ausnutzung von Gefühlen unmerklich in eines der Menschenwürdeverletzung zu überführen, ohne seinen Gehalt zu verändern. Ansätze dazu gab es bereits in der ersten BGH-Entscheidung, in der das Ausnutzungsargument zwar im Vordergrund der Argumentation stand, in der der BGH die Menschenwürde HIV-positiver Personen jedoch auch und gerade durch die »zynische und menschenverachtende« Verbindung mit kommerziellen Interessen betroffen ansah. ${ }^{44}$ Das eigentliche Sachargument bleibt also unverändert: In beiden Entscheidungen betont der BGH die Besonderheit des (kommerziellen) Werbekontextes.45 Unterstützend führt er die Interessen und

Direktiven in Generalklauseln. Möglichkeiten und Grenzen der empirischen Sozialforschung bei der Präzisierung der Gute-Sitten-Klauseln im Privatrecht, I97I. Auch im »Busengrabscher «-Urteil stellte der BGH neben den normativen Begriff des »Anstandsgefühls « die empirische Missbilligung eines Verhaltens durch die Allgemeinheit als untragbar (BGH, I8. 5. I995, BGHZ I30, S. sff. (S. 7)). In diesem Urteil werden jedoch in der Darstellung empirische und normative Feststellungen klar getrennt. Ausführlich äußert sich zum Anwendungsbereich empirischer Argumente und ihrer Verifizierung das Senatsmitglied Ullmann, GRUR i99i, S. $789 \mathrm{ff}$.

40 Darauf weist Ahrens (Fn. I 5), S. 1098 hin.

4I So auch die Kritik von Hoffmann-Riem, Kommunikationsfreiheit für Werbung - zugleich Anmerkung zu den Benetton-Entscheidungen des BGH, ZUM I996, S. 6 ff.; S. I I.

42 Der Umgang mit diesen wird kritisiert zum Beispiel von Hoffmann-Riem (Fn. 4I), S. I I f., der auf anders lautende öffentliche Stellungnahme Betroffener verweist; dazu auch kritisch Sevecke (Fn. I4), S. I43 f.

43 BGH, 6. I 2. 200I, BGHZ I 49, S. 247 ff. (S. 265 f.).

44 BGH, 7.6. 1995, NJW I995, S. 2492 f.

45 In diesem Zusammenhang versäumt er auch durchgängig eine Unterscheidung zwischen Schutzbereich und Schranken (in deren Rahmen der Werbekontext durchaus eine Rolle hätte spielen dürfen); siehe so vor 
Gefühle von Wettbewerberinnen und Wettbewerbern an, die durch die Zwänge des Wettbewerbs zu vergleichbaren Werbemethoden gezwungen werden könnten. ${ }^{46}$ Da das zentrale Argument des BGH also unverändert geblieben war, konnte es nicht überraschen, wenn das Bundesverfassungsgericht jetzt auch dieses Urteil wieder aufgehoben hat: Trotz des Werbekontextes und des Verzichts auf einen eigenen Kommentar veranschauliche das Bild einen gesellschaftlichen Missstand und habe meinungsbildenden Inhalt. Zwar könne der Werbezweck den Aussagegehalt einer Botschaft beeinflussen. Der Werbekontext verändere aber nicht den Maßstab, der an eine meinungsbildende Maßnahme anzulegen sei. ${ }^{47}$ Die Schlusssätze lauten: »Wollte man kommerziellen Werbeanzeigen wegen des mit ihnen stets verbundenen Eigennutzes die Thematisierung von Leid verbieten, hätte ein wesentlicher Teil der Realität in der allgegenwärtigen, Sichtweisen, Werte und Einstellungen der Menschen nicht unerheblich beeinflussenden Werbewelt von vornherein keinen Platz. Das kann angesichts des besonders schützenswerten Interesses an der Thematisierung gesellschaftlicher Probleme kein mit der Meinungs- und der Pressefreiheit vereinbares Ergebnis sein.«

\section{IV. Öffentliche Kommunikation und private Interessen}

\section{Werbung als Politik?}

Damit ist diese Form der Werbung als Akt öffentlicher Kommunikation nicht nur dem Schutzbereich der Kommunikationsgrundrechte zugeordnet, sondern auch denselben Maßstäben unterworfen wie die öffentliche Kommunikation mit ausschließlich politischen Zielsetzungen. Allerdings gilt dies nur für eine ganz bestimmte Form der Werbung, und insofern ist die verfassungsgerichtliche Rechtsprechung tatsächlich spezifisch auf eine neuartige Form der Werbung bezogen. Nicht jede Verbindung von kommerziellen Produkten mit »Gefühlen« (wie sie z.B. auch in der Propagierung eines Likörs als Mittel zur Überwindung sexueller Widerstände stattfindet ${ }^{4}$ ) unterfällt dem Schutzbereich des Art. 5 GG. Die Kommunikation über Gefühle ist der Werbung seit jeher eigen und insofern eine ungeeignete Kategorie. Voraussetzung für den Grundrechtsschutz ist eine inhaltliche Intervention im öffentlichen Raum. ${ }^{49}$ Und genau darin liegt auch die Neuartigkeit dieser Form der »Werbung« bzw. »Öffentlichkeitsarbeit« privatwirtschaftlicher Unternehmen. Als » Werbung mit der Reali-

allem Hoffmann-Riem (Fn. 4I), S. 2; Sevecke (Fn. I4), S. I I 8 ff.; Schulze-Fielitz (Fn. I 5), S. 302; Wassermeyer (Fn. 27), S. I33; vgl. auch Schulze-Fielitz (Fn. I 5), S. 304 mit einer Kritik an der Verwendung des Menschenwürdearguments als »kleiner Münze«.

46 BGH, 6. I 2. 200 I, BGHZ I49, S. 247 ff. (S. 27 I f.). Zur »Aufschaukelungsgefahr« ausführlich Kassebohm (Fn. 29), S. $29 \mathrm{ff}$.

47 Auf diesen Unterschied geht vor allem More Disagreement Over Human Dignity: Federal Constitutional Court's Most Recent Benetton Advertising Decision, 6 German Law Journal Vol. 4 (2003), www.germanlawjournal.com, ein.

48 BGH, I 8. 5. 1995, BGHZ I30, S. 5 ff. (S. 8 f.) (»Busengrabscher«); siehe auch BVerfG, Kammerbeschluss vom 27. 5. I994, NJW I 994, S. 3342 f. (»Mars-Kondom«).

49 Zum Umfang des Schutzbereichs siehe insofern auch BVerfG, I. 8. 200I, NJW 200I, S. 3403 ff. (»reine Wirtschaftswerbung, die einen wertenden, meinungsbildenden Inhalt hat «); Schulze-Fielitz (Fn. I 5), S. 303 hält den Schutzbereich (wohl weiter gehend als das BVerfG) bei jedwedem »informativ-wertenden« Zweck für einschlägig. Sevecke (Fn. I4), S. 26 f. zieht deshalb zu Recht Parallelen zu den Togal- und Tengelmann-Werbungen aus den Jahren I 993 und I994, in denen Kritik an PolitikerInnen geübt bzw. Wahlwerbung gemacht wurde; ders., S. I I 8 ff. zum Schutzbereich des Art. s Abs. I Satz I GG für Werbende. Das Togal-Urteil des BGH vom I 5. 5. 1997 wurde veröffentlicht zum Beispiel in NJW 1998, S. 604 ff. (der BGH und das OLG München hielten die Werbung im Gegensatz zum LG München weitgehend für zulässig). 


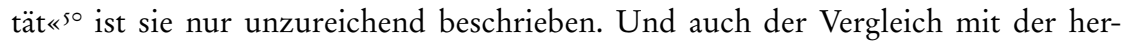

kömmlichen Unterscheidung zwischen Werbung und Public Relations als verschiedenen Formen des Marketing, für die verschiedene Maßstäbe gelten müssten, geht fehl. ${ }^{\text {I }}$ Das Besondere ist hier nicht, dass Formen bewusst und gewollt vermischt werden, sondern dass die Kommunikation als öffentliche Kommunikation ernst gemeint ist und verstanden wird. Zu Recht verweist das Bundesverfassungsgericht darauf, dass sie nur unter dieser Voraussetzung überhaupt die vom Bundesgerichtshof dargelegten Wirkungen erzeugen könne. ${ }^{52}$

Solche Formen der Werbung, die gleichzeitig ernst gemeinte Interventionen darstellen, sind aber nur deshalb möglich, weil sich auch die Formen der politischen Kommunikation verändern und verändert haben. So konnten Veranstaltungen wie die »Love Parade« als politische Demonstrationen bzw. Versammlungen im Sinne einer kollektiven Meinungskundgabe präsentiert werden.53 So ist in der diesjährigen Antikriegs- und Friedensbewegung die Grenze zwischen Life-Style-Bewegung, Jugendbewegung und politischer Bewegung fließend geworden. Die Abzeichen der Friedensbewegung werden jedenfalls weithin auch als Mode-Accessoires eingesetzt. Und andere soziale Bewegungen setzen statt auf die politische Regulierung zunehmend auf direkte Ansprache der Unternehmen und die Etablierung von Sozial- und Umwelt»Labels « oder-zertifikaten als Produktkennzeichen. ${ }^{44}$ Politisch mag man diese Form der Kopplung zwischen Politik und Wirtschaftssystem kritisieren und auf eine Wiederbelebung genuin politischer Codes dringen; das autoritative (wettbewerbs-) rechtliche Verbot ist aber sicher nicht das richtige Mittel, um dieser Entwicklung Einhalt zu gebieten. Will man die Verwendung politischer Kommunikation in kommerziellen Zusammenhängen beschränken, muss man die Verfügungstellung öffentlicher Räume für privatwirtschaftliche Zwecke grundsätzlich diskutieren dann aber gerade auch für die herkömmliche Werbung mit Frauen, Sex und Familienglück.5s

50 Sosnitza (Fn. 49), S. 540 .

5 I Henning-Bodewig (Fn. 25), S. 952 versuchte, unterschiedliche Maßstäbe für Public-Relations-Maßnahmen und »tradierte Werbemethoden « zu entwickeln. Zur Unterscheidung von unternehmensbezogener Imagewerbung und produkt- und dienstleistungsbezogener Werbung siehe auch Cordes, Umweltwerbung. Wettbewerbsrechtliche Grenzen der Werbung mit Umweltschutzargumenten, I 994, S. 9 ff.; genauer zur unternehmensbezogenen (Umwelt-)Werbung S. I4 ff. Vgl. auch Fezer (Fn. I2), S. 582: Man könne nicht bestimmte Werbestrategien verlangen.

52 Deshalb kann auch das Gewicht der wirtschaftlichen und der politischen Bedeutung einer Maßnahme Kriterium der Abwägung und der Frage der Rechtmäßigkeit eines Eingriffs in die Meinungsfreiheit sein (Fezer (Fn. I 2), S. 58 I; dort auch genauer zur Entwicklung der Werbung von der persuasiven Reklame zur informativen Kommunikation). Hoffmann-Riem (Fn. 4I), S. 2; er verweist, S. 3, auch darauf, dass Luciano Benetton auch selbst aktiver Politiker ist.

53 Die »Love Parade« wurde letztlich (im Gegensatz zur »Fuck Parade«) durch das VG Berlin (25. 6. I999, LKV I 999, S. 373 ff.) und das OVG Berlin (8. 7. I999, LKV I999, S. 372 f.) als nicht dem Schutzbereich des Art. 8 GG unterfallend behandelt, was die r. Kammer des BVerfG mit Einstweiliger Anordnung vom I 2. 7. 200I, NJW 200I, S. 2459 ff. akzeptierte; dies hatte zur Konsequenz, dass für Verkaufsstände Sondernutzungserlaubnisse auch an Dritte, kommerzielle AnbieterInnen erteilt wurden, was die Veranstalter des »Love Parade Forum « als Aufdrängung eines kommerziellen Gepräges kritisierten; zur Diskussion siehe auch Wiefelspütz, Ist die Love-Parade eine Versammlung?, NJW 2002, S. 274 ff.; Deger, Sind Chaos-Tage und Techno-Paraden Versammlungen?, NJW I 997, S. 923 ff. Siehe auch zu diesen Veränderungen Axford/ Huggins, Political Marketing and the Aestheticisation of Politics: Modern Politics and Postmodern Trends, in: O’Shaughnessy/Henneberg (Hrsg.), The Idea of Political Marketing, 2002, S. I 92 f.

54 Einen Überblick bietet die Verbraucher Initiative e. V. auf www.label-online.de.

55 Fezer (Fn. 49), S. 275 (der sich durch die ständige Begegnung mit dem »Marlboro«-Cowboy stärker belästigt fühlt als durch Benettons Darstellung einer ölverschmutzten Ente). Der Verwaltungsratschef des Bundesverbandes der Verbraucherzentralen, Rips, wies auf der 50-Jahr-Feier für den Verbraucherschutz am 5. 6. 2003 auch auf die öffentliche Subventionierung der Werbung in Form der steuerlichen Absetzbarkeit hin. 
Mit seiner Bewertung der meinungsbildenden Funktionen bestimmter Formen kommerzieller Werbung weist das Bundesverfassungsgericht somit auf einen Wandel im Verhältnis von Wirtschaft, Politik und Gesellschaft hin. Leider benennt es dies selbst nicht. In seiner ersten Benetton-Entscheidung vergleicht es die »H.I.V. positive«Plakate mit einem »Großteil heutiger Werbung «, die zum Beispiel Prominenz oder »das Versprechen sportlicher Anerkennung « als Kaufanreiz einsetze und die vom BGH in der Regel nicht beanstandet werde (als Beispiel hätte auch darauf verwiesen werden können, dass das obligatorische Frauen-Model(l) in vielen Werbungen präsenter ist als das beiläufig platzierte Produkt). »Produktunabhängige Imagewerbung hat sich eingebürgert, ohne dass der Leistungswettbewerb darunter erkennbar gelitten hat $\ll .{ }^{6}$

Noch deutlicher als hier hat das BVerfG in der Kammerentscheidung, mit der Urteile des BGH zur Werbung mit Tierschutzargumenten als offensichtlich verfassungswidrig aufgehoben wurden, den »Leistungswettbewerb « als ein schützenswertes Rechtsgut bezeichnet. Es vermisste insofern in den aufgehobenen Urteilen lediglich eine konkrete Darstellung, inwiefern der Leistungswettbewerb durch die verbotene Werbung gefährdet sei; schließlich wollten die Anbieter hier über die Angaben zu Preis und Qualität hinaus durch »weitere Informationen « zum Kauf motivieren. ${ }^{57}$ In der Literatur wurde darüber hinaus darauf hingewiesen, dass es keine empirische Evidenz von »Abstumpfungstendenzen « gebe. Möglicherweise werde durch die Thematisierung sozialer Probleme das Bewusstsein der Öffentlichkeit sogar geschärft, was bei herkömmlichen Werbeformen, die zum Konsum »erziehen«, gerade ausgeschlossen sei. ${ }^{8}$

Diese Hinweise enthalten natürlich implizit den Vorwurf, das Sittenwidrigkeitsgefühl des BGH werde mehr von einer Anstands- und Sozialmoral angetrieben, für die eine Auseinandersetzung mit gesellschaftlichem Elend Privatsache ist, 59 als von dem Anliegen, Abstumpfung und Diskriminierung zu verhindern - würde ein solches Anliegen ernsthaft verfolgt, wäre der PORNO-Kampagne in den 8 oer Jahren vielleicht tatsächlich schon mehr Erfolg vor den Zivilgerichten beschieden gewesen. ${ }^{60} \mathrm{Mit}$ diesen Argumenten des »das machen doch alle« und »sonst habt ihr doch auch nichts dagegen « vergibt das Bundesverfassungsgericht aber auch die Chance, die Unterschiede der Benetton-Kampagne zu herkömmlichen Werbeformen deutlich zu machen. Dies wäre aber erforderlich, um sich darüber klar zu werden, welche Fallgruppen und welche Kategorien der sittenwidrigen Werbung in der Rechtsprechung des BGH nun zur Debatte stehen müssen.

Jedenfalls die Rechtsprechung des BGH zur Umweltwerbung und zum Social Sponsoring wird nicht mehr zu halten sein; ${ }^{61}$ wahrscheinlich wird darüber hinaus die gesamte

56 BVerfG, I 2. I 2. 2000, BVerfGE I02, S. 347 ff. (S. 364 ).

57 BVerfG, Kammerentscheidung vom I. 8.2001, NJW 2001, S. 3403 ff.

58 Sosnitza (Fn. 49), S. 544 f.; vgl. auch Fezer (Fn. 49), S. 275. Ahrens (Fn. I 5), S. I099 sieht bezeichnender Weise Abstumpfungstendenzen eher als Gefahr der Herstellung eines werbefeindlichen Klimas.

59 Vgl. die in Fn. 29 zitierten Annahmen.

60 Dazu Baer/Slupik, Entwurf eines Gesetzes gegen Pornographie, KJ i988, S. I7 I ff. mit einem dem UWG strukturell verwandten Gesetzentwurf; kritisch Renk, Zivilrecht als Instrument gegen Sexismus?, KJ I 988 , S. I 82 ff.; für eine entsprechende Klausel in einem Antidiskriminierungsgesetz schon Coester-Waltjen, Zielsetzung und Effektivität eines Antidiskriminierungsgesetzes, ZRP I982, S. 22 I; siehe aber auch jetzt BGH, I8. 5. 1995, BGHZ I30, S. 5 ff. (»Busengrabscher«); zu den Veränderungen auch Steinbeck, Das Bild der Frau in der Werbung, ZRP 2002, S. $435 \mathrm{ff}$.

6I Fezer (Fn. I2), S. 582; rechtspolitisch Fezer, Modernisierung des deutschen Rechts gegen den unlauteren Wettbewerb auf der Grundlage einer Europäisierung des Wettbewerbsrechts, Gutachten für das BMJ vom I 5. 6. 200 I (http://www.bmj.bund.de/images/ı09I9.pdf), S. 88 ff.; zur Parallele des Sponsoring vor allem Hoffmann-Riem (Fn. 4I), S. 4; er weist, S. ıо, darauf hin, dass das Rundfunkrecht sogar eine Pflicht zur 
die UWG-Reform vor. ${ }^{63}$ Hier geht es im Kern nicht um den Gegensatz zwischen dem Leitbild eines flüchtigen Verbrauchers und dem eines verständigen Verbrauchers. ${ }^{64}$ Hier geht es vielmehr darum, dass der BGH von einem anderen Gegenstand von Verbraucherentscheidungen ausgeht und damit von einem anderen Werbe- und Wettbewerbsverständnis, als es sich in der Benetton-Kampagne dokumentiert: Mit der Aufhebung der BGH-Urteile wird fraglich, inwieweit nicht die Kategorie des »Leistungswettbewerbs « als Auslegungsdirektive für das Vorliegen von Sittenwidrigkeit und Unlauterkeit insgesamt in Frage zu stellen ist. Denn diese Formel, die im Wettbewerbsrecht seit Längerem kritisiert wird, ist immer noch wichtiger Bestandteil der wettbewerbsrechtlichen Dogmatik nicht zuletzt der zivilrichterlichen Rechtsprechung. ${ }^{65}$ Dort wird sie in der Regel mit dem Sachlichkeitsgrundsatz verbunden. ${ }^{66}$ Die Rechtswissenschaft geht insofern nach wie vor davon aus, dass Werbung Verhalten und Entscheidungen unmittelbar und rational beeinflussen solle - im Gegensatz zur sozial- und betriebswirtschaftlichen Werbetheorie, in der zunächst die Positionierung von Produkten betont wird; eine informierte interdisziplinäre Betrachtung hätte motivationspsychologische Erkenntnisse zur Wirkung von Werbung und speziell der life-style-Werbung einbeziehen können. ${ }^{67}$ Natürlich könnte auch lifestyle-Werbung unter einen weiten Begriff des Leistungswettbewerbs gefasst werden; auch der Image-Transport und mögliche Distinktionsgewinne der Käuferinnen und Käufer können als Leistungen der Produkte gesehen werden ${ }^{68}$ - die bei zunehmendem »Qualitätspatt« von Produkten zunehmend in den Vordergrund treten. ${ }^{69}$ Der Begriff wird damit aber seines bisherigen sachlichen Gehalts weitgehend entleert.

Nennung des Sponsors vorsieht; siehe auch die Parallele zum Product Placement, die Sevecke (Fn. I4), S. 52 f. erwähnt.

62 So auch Hartwig, Verfassungsrechtliche Anforderungen an die Fallgruppenbildung nach $₫$ I UWG, NJW 2002, S. 39.

63 So insbesondere das vorbereitende Gutachten von Fezer (Fn. 6I), S. 90. Der Regierungsentwurf eines Gesetzes gegen den unlauteren Wettbewerb laut Kabinettsbeschluss vom 7.5. 2003, www.bmj.bund.de/ images/ I I 596.pdf geht nicht ausdrücklich darauf ein; die Fallgruppen in $\$ 4$ setzen jedenfalls entweder Beeinträchtigung der Entscheidungsfreiheit (Nr. I) oder Verschleierung des werblichen Charakters (Nr. 4) voraus; die Fallgruppe der Belästigung (Nr. 3 ) wiederum stellt eher auf persönlich adressierte und versandte Werbung ab.

64 Das deutet Fezer (Fn. 6I), S. I3 an.

65 So betont der BGH im zweiten Benetton-Urteil vom 6. I 2.200I, dass es darum gehe, Auswüchse des Wettbewerbs zu bekämpfen, die dazu beitragen können, »den Wettbewerb zu vergiften, und einen an der Leistung orientierten Wettbewerb gefährden «; am Ende wird der Schutz des Leistungswettbewerbs als eins der Schutzgüter des UWG noch einmal betont. Der Begriff wird zum Beispiel von Henning-Bodewig (Fn. 29), S. 539 im Sinne des BGH, und von Bülow (Fn. 7) S. I 289 ff. auch gegen die BGH-Rechtsprechung verwandt. Genauer auch Baumbach/Hefermehl (Fn. 6), Allg, Rn 22; Einl UWG, Rn 96 ff. (Rn Ios: kein normatives Merkmal, aber Indiz für die Sittenwidrigkeitsbeurteilung). Der »aktuelle Stand der Diskussion im Wettbewerbsrecht«, demzufolge es sich hierbei um eine Leerformel handele (Hartwig (Fn. 62), S. 39), hat sich also noch nicht in der zivilgerichtlichen Rechtsprechung durchgesetzt. Auch Fezer (Fn. 6I), S. 28 benennt den Schutz des Leistungswettbewerbs als Schutzgut eines »modernen Wettbewerbsrechts«; ausführlich S. 3 I f. zum »Leistungswettbewerb « als Auslegungsdirektive. Siehe auch die Kritik an dem »diffusen Begriff « bei Busche, LM Nr. $866 \mathrm{zu} \ \mathrm{I}$ UWG.

66 Auch für Henning-Bodewig (Fn. 29), S. 536 trägt gerade die fehlende Sachlichkeit zur Schockwirkung bei; ähnlich Kassebohm (Fn. 29), S. I45; vgl. Wassermeyer (Fn. 27), S. I 27 ff. Andere weisen darauf hin, dass umgekehrt gerade bei einem Produktbezug die Sittenwidrigkeit eher zu bejahen sei (siehe zum Beispiel Hoffmann-Riem (Fn. 4I), S. 2 ff.); siehe auch die Äußerung der Benetton-Sprecherin Marina Galanti: »That seems to mean that if we had been selling bulletproof vests it would have been OK to use the picture of the Croatian soldier « (zitiert bei Kassebohm (Fn. 29), S. 86); vgl. auch $\ 4$ Nr. I des Regierungsentwurfs eines Gesetzes gegen den unlauteren Wettbewerb laut Kabinettsbeschluss vom 7.5.2003, www.bmj.bund.de/images/ i i $596 . p d f$.

67 Siehe dazu zum Beispiel Bergler/Pörzgen/Harich (Fn. 2), S. 38 ff.; auch Sevecke (Fn. I4), S. 32 ff. und vor allem S. 93 ff. geht auf alle »Kommunikationsfunktionen« der Werbung ein. Für die rechtswissenschaftliche Nutzung betriebswirtschaftlicher Theorien unter Betonung der Verhaltensbeeinflussung siehe Kassebohm (Fn. 29), S. 23 ff. m.w. N.

68 Bülow (Fn. 7), S. I 290; Kassebohm (Fn. 29), S. 97 ff.; S. I05.

69 Bruhn/Homburg (Fn. 2), »Positionierung«. 
Die Debatte zeigt damit die Fragwürdigkeit eines Lauterkeitsverständnisses auf, mit dem bestimmte Inhalte wettbewerblichen Handelns und unternehmerischer Leistung vorgegeben werden. Daran, dass er die Schutzgüter seines Begriffs des »Leistungswettbewerbs « als Gegenstand der »Lauterkeit « nicht benennen konnte, ist der BGH in der Auseinandersetzung mit dem Bundesverfassungsgericht letztlich gescheitert. Der Begriff der » Leistung « kann als Leerformel für die Ergebnisse unternehmerischen Handelns auf dem Markt nur funktionieren, wenn er offen gehalten wird für Erweiterungen des Produktbegriffs (zum Beispiel in Richtung von Dimensionen der »Sozialverträglichkeit «, der Nachhaltigkeit und performativer Aussagen). $7^{\circ}$

\section{Rechtsgüterschutz im Wettbewerb}

Künftig wird vor dem Hintergrund solcher Leerformeln vor allem auszuloten sein, wie Lauterkeit in der Werbung weniger positiv als vielmehr negativ über den Begriff der Unlauterkeit und der Verletzung geschützter Rechtsgüter bestimmt werden kann. So hat in Fällen von Meinungsäußerungen in Form von Boykottaufrufen als Grenze der Grad der Beeinträchtigung konkreter wirtschaftlicher Interessen der Konkurrentinnen und Konkurrenten gedient. Danach bedarf es jeweils einer Interpretation konkreter Wirkungen und Interessen. ${ }^{71}$

Eine weitere Grenze der politischen Kommunikation im Wettbewerb ist das Irreführungsverbot. ${ }^{72}$ Selbst eine objektiv richtige Angabe sieht die Zivilrechtsprechung als irreführend an, wenn ein nicht völlig unerheblicher Teil der umworbenen Verkehrskreise damit eine unrichtige Vorstellung verbindet.73 Bei der Werbung mit Umweltschutzargumenten geht der BGH davon aus, dass ihr ein hohes Irreführungspotenzial $\mathrm{zu}$ eigen sei; dies hänge mit dem hohen Umweltbewusstsein der Bevölkerung bei schwer vermittelbaren komplexen Sachzusammenhängen und einer weit gehenden Unklarheit über Bedeutung und Inhalt der Begriffe »umweltfreundlich«, »bio« u. ä. zusammen. ${ }^{74}$ Ein Zeichen, Label oder Gütesiegel kann damit zurzeit im Regelfall nur dann ohne Kommentar verwendet werden, wenn die Vergabebedingungen des Zeichens in der beworbenen Gruppe bekannt sind 75 oder sonstwie deutlich gemacht wird, worauf die Verleihung des Zeichens beruhe. ${ }^{76}$ Hier wäre künftig darauf zu achten, dass der tatsächliche Aussagegehalt dieser Labels tatsächlich nach einem

70 Bei den Fallgruppen des »Leistungsschutzes « geht es hingegen um etwas anderes, nämlich um den Schutz vor Imitation und Nachahmung (genauer Fezer (Fn. 6I), S. 50 ff.); vgl. dort S. 59 f. auch zur Erweiterung des Leistungsinhalts unternehmerischer Produkte; siehe auch die Ersetzung des Begriffs der Sittenwidrigkeit durch den der Unlauterkeit im Regierungsentwurf eines Gesetzes gegen den unlauteren Wettbewerb laut Kabinettsbeschluss vom 7.5.2003, www.bmj.bund.de/images/i I $596 . p d f$.

7I Zum Beispiel wieder BVerfG, Kammerbeschluss vom 4. I0. I988, NJW I992, S. I I 53 f. und im Ergebnis bestätigend EGMR, 23.6. 1994 (Jacubowski ./. Germany) NJW I995, S. 857 f. mit abweichenden Meinungen. Siehe hierzu schon die Lüth-Entscheidung des BVerfG, I5. I. I958, BVerfGE 7, S. $198 \mathrm{ff}$.

$72 \mathrm{Vgl} . \$ 5$ des Regierungsentwurfs eines Gesetzes gegen den unlauteren Wettbewerb laut Kabinettsbeschluss vom 7.5. 2003 , www.bmj.bund.de/images/ I I 596 .pdf.

73 Baumbach/Hefermehl (Fn. 6), \$3 UWG, Rn 25 ff.

74 Baumbach/Hefermehl (Fn. 6), \ I UWG, Rn I79a ff.; Cordes (Fn. 51), S. 91; BGH, 5. I 2. I996, DB I997, S. 2 I I9f; genauer zum Bedeutungsgehalt umweltbezogener Werbeangaben S. 98 ff.; BGH 20. I0. I988, GRUR I99I, S. 548 ff. Mit dieser Begründung wurde zum Beispiel im Grundsatzurteil »Umwelt-Engel « dem Einzelhandelsunternehmen Kaiser's Kaffee untersagt, sein Öko-Sortiment mit dem »Blauen Engel« zu markieren: BGH, 20. I0. I988, BGHZ I05, S. 277 ff.; BGH, 5. I2. I996, DB I997, S. 2 I I9f.; auf mangelnde Transparenz stellte auch die Krombacher-Entscheidung des OLG Hamm, I 2. II. 2002, NJW 2003, S. 1745 ff. ab; zum Vergabeverfahren des »Blauen Engels« genauer Cordes (Fn. 5i), S. 29 ff.; siehe auch zum Irreführungsaspekt einer Werbung mit Testergebnissen der Stiftung Warentest Horn/ Piepenbrock (Hrsg.), Vergleichender Warentest: Testpraxis, Testwerbung, Rechtsprechung, 986.

75 Cordes (Fn. 5I), S. I 27 ff.; zu "privaten« Zeichen S. I4 I ff.

76 Baumbach/Hefermehl (Fn. 6), \I UWG, Rn I 8 Ib. 
empirisch feststellbaren Empfängerhorizont ermittelt wird (wobei es dann durchaus eine Rolle spielt, an welchem Verbraucherleitbild man sich orientiert).

Die Orientierung am Irreführungsverbot führt auf eine allgemeine Orientierung an der Markttransparenz als eines Lauterkeitsinteresses der Allgemeinheit. ${ }^{77}$ Es hat durchaus auch schon Ansatzpunkte dafür gegeben, die Zielsetzung des Wettbewerbsrechts, »verlässliche Orientierungen für verantwortungsbewusste Konsumentscheidungen « zu schaffen, durch gesetzliche Vorschriften zu unterstützen. Entgegen ersten Plänen der Bundesregierung sind nun aber weder im Entwurf eines Verbraucherinformationsgesetzes noch im UWG-Reformentwurf Auskunfts- und Informationsansprüche von Verbraucherinnen und Verbrauchern gegenüber Unternehmen vorgesehen. ${ }^{78}$

Darüber hinaus wird künftig die Fallgruppe der diskriminierenden Werbung genauer herauszuarbeiten sein.79 Auf die zivilgerichtliche Bewertung von Werbemaßnahmen, die Frauen, Behinderte oder Minderheiten herabsetzen, ist im Zusammenhang mit den Benetton-Urteilen nicht zuletzt deshalb oft verwiesen worden, weil an ihr Diskrepanzen in der Bewertung verschiedener »Gefühle« durch die Zivilrechtsprechung illustriert werden konnten: Solidarische Gefühle des Mitleids sind zum Teil von der Zivilgerichtsbarkeit offensichtlich als für das rationale Verbraucherhandeln gefährlicher eingeschätzt worden als Gefühle der Verachtung. In Bezug auf Letztere hat sich jedoch schon vor der Benetton-Entscheidung ein Wandel angedeutet; ${ }^{8 \circ}$ dem Bundesverfassungsgericht kommt darüber hinaus das Verdienst zu, auf die Funktionen kommerzieller Werbung in der öffentlichen Kommunikation aufmerksam gemacht zu haben.

77 Zum Wandel des Wettbewerbsrechts von subjektiv- zu objektivrechtlichen Maßstäben Fezer (Fn. 6r), S. 23 ff.; vgl. zum Gesichtspunkt der Markttransparenz im hier besprochenen Zusammenhang auch Sevecke (Fn. I4), S. 32 ff.; S. 8 I.

78 Ausführlich zu diesen Möglichkeiten sowie der Unterstützung von Siegeln mit hohem Bekanntheitsgrad und breitem Anwendungsbereich Günther, Verbraucherinformation - mehr als Zugangsrechte. Denkanstöße für eine verstärkt konzeptionelle Ausrichtung der Verbraucherpolitik, VuR 2003, S. 29 ff. Der Gesetzentwurf für ein Verbraucherinformationsgesetz vom 8.3.2002 mit Begründung findet sich auf www.verbraucherministerium.de/verbraucher/verbraucherinfos.htm. Es enthält nur noch Auskunftsansprüche gegen Behörden bzw. Private, die öffentliche Aufgaben wahrnehmen. Regierungsentwurf eines Gesetzes gegen den unlauteren Wettbewerb laut Kabinettsbeschluss vom 7.5.2003, www.bmj.bund.de/ images/I I 596.pdf; zur Kritik zum Beispiel Presseerklärung des Verbraucherzentrale Bundesverband e. V. vom 8.5. 2003 (auf www.vzbv.de).

79 Fezer (Fn. I2), S. 582; zu den Fallkonstellationen der rassen-, ausländer-, religionen-, behinderten- und geschlechterdiskriminierenden Werbung Fezer (Fn. 49), S. 265 (S. 272 ff.); rechtspolitisch auch Fezer (Fn. 6I), S. 86 ff. und genauer zu den Fallgruppen S. 90 ff.; auch der Bundesverband der Verbraucherzentralen vzbv schlägt eine ausdrückliche Normierung dieser Fallgruppen in der UWG-Reform vor (siehe vzbv, Stellungnahme vom 28.5.2003, www.vzbv.de/mediapics/stellungnahme_regierungsentwurf_ uwg_203.pdf); genauer zur geschlechter-(frauen-)diskriminierenden Werbung Bergler/Pörzgen/Harich (Fn. 2); Hering, Weibsbilder. Zeugnisse zum öffentlichen Ansehen der Frau, 2. Aufl. 1987; Steinbeck (Fn. 60).

80 BGH, I 8. 5. 1995, BGHZ I 30, S. 5 ff. (»Busengrabscher«). 